Original Article

\title{
A STUDY ON OCCURRENCE OF SELECTED RISK FACTORS OF PREGNANCY AMONG ANTENATAL WOMEN WITH A VIEW TO DEVELOP AN INFORMATION BOOKLET
}

\author{
Neethu Varghese ${ }^{1} \&$ Philomena Fernandes ${ }^{2}$ \\ ${ }^{1}$ Lecturer, Department of Obstetrics \& Gynaecological Nursing, Anjarakandy Medical College, Kannur, Kerala, \\ ${ }^{2}$ Associate Professor \& HOD, Department of Obstetrics \& Gynaecological Nursing, \\ Nitte Usha Institute of Nursing Sciences, Nitte University, Mangalore, Karnataka, India.

\section{Correspondence: \\ Philomena Fernandes} \\ Associate Professor \& HOD, Department of Obstetrics \& Gynaecological Nursing, Nitte Usha Institute of Nursing Sciences, Nitte \\ University, Mangalore - 575 018, Karnataka, India. \\ Mobile: +919449207845 E-mail : philferns7@gmail.com
}

\begin{abstract}
:
The descriptive study was conducted to determine the occurrence of selected risk factors of pregnancy among antenatal women. Structured interview schedule was used to assess the risk factors of pregnancy among antenatal women. 150 samples were selected by purposive sampling technique. The study findings revealed that increased maternal age, short stature, increased blood pressure, abortion, decreased hemoglobin and GDM were the most prevalent risk factors in the study sample. There was significant association between gravid state and abortion (cal=26.78, $p<0.05$ ), gravid state and age (cal=9.79, $p<0.05$ ), education and hemoglobin level (cal=6.02, $p<0.05$ ) at 0.05 level of significance.
\end{abstract}

Keywords: risk factors, antenatal women, information booklet

\section{Introduction:}

Pregnancy is a unique, exciting and often joyous time in a woman's life, as it highlights the woman's amazing creative and nurturing powers while providing a bridge to the future. However, a pregnant woman needs to be a responsible woman so as to best support the health of her future child, as the growing fetus depends entirely on its mother's healthy body for all needs1. Pregnancy is a natural physiological process but there are certain high risk factors that may come across and complicate the pregnancy and childbirth and often pregnant women are not aware of the seriousness of these problems.

According to $\mathrm{WHO}$, worldwide maternal mortality ratio is

\begin{tabular}{|c|}
\hline Access this article online \\
\hline Quick Response Code \\
\hline
\end{tabular}
estimated to be 400 per 1 , 00,000 live births with 5 , 36,000 maternal deaths occurring annually and global lifetime risk of death from pregnancy of 1 in 92(2009). In India currently (2010) MMR is found to be 290 per 1, 00,000 live births. Identification of the risk factors together with appropriate and timely interventions during the perinatal period can prevent morbidity and mortality among mothers and infants to a great extent. For health professionals maternal mortality is not statistics, not rates or ratios but it is women whose faces are seen in the throes of agony, distress and despair, and this is not simply because these women die in the prime of their lives, at a time of great expectation and joy or not because maternal death is one of the most terrible ways to die, be it bleeding to death, the convulsions of toxaemia of pregnancy, the unbearable pangs of obstructed labour or the agony of puerperal sepsis. It is because in almost each and every case, it is a 5 event that could have been prevented. So the investigator feels that nurses are responsible and accountable for early identification of risk factors and also imparting health education to gravid mothers for prevention of this enduring epidemic.

\section{Objectives of the Study \\ $\infty$ To determine the occurrence of selected risk factors of}


pregnancy among antenatal women.

$\infty$ To develop an information booklet on selected risk factors of pregnancy

$\infty$ To find the association of selected risk factors of pregnancy among

$\infty$ antenatal women with selected demographic variables

\section{Materials and Methods:}

The study adopted a survey approach with a descriptive design and was conducted in Justice K.S Hegde Charitable Hospital Mangalore from 16/8/11 to 25/9/11. For the study 150 antenatal women were selected through purposive sampling technique. An informed consent was taken from all subjects and data were collected by structured interview schedule on selected risk factors of pregnancy. The selected risk factors were categorised into biophysical factors, psychosocialfactors and environmental factors. Biophysical factors includes age, height, various medical disorders and obstetrical factors. Psychosocial factors includes history of psychiatric disorders, smoking or alchohol, access to health care facilities, support from family or spouse. Environmental factors includes exposure to radiation, pesticides or industrial pollutants. The data was analysed using descriptive and inferential statistics.

Results:

Major findings are discussed under the following headings

Section 1: Description of sample characteristics

$n=150$

\begin{tabular}{|c|c|c|c|}
\hline $\begin{array}{l}\text { SL. } \\
\text { NO }\end{array}$ & $\begin{array}{l}\text { DEMOGRAPHIC } \\
\text { VARIABLE }\end{array}$ & $\begin{array}{l}\text { FREQUENCY } \\
\text { (f) }\end{array}$ & $\begin{array}{c}\text { PERCENTAGE } \\
(\%)\end{array}$ \\
\hline 1 & Age of the mother & & \\
\hline a & 18-23yrs & 40 & 26.7 \\
\hline $\mathrm{b}$ & 24-29yrs & 76 & 50.6 \\
\hline C & $30-35$ yrs & 28 & 18.7 \\
\hline $\mathrm{d}$ & $36-41$ yrs & 6 & 4 \\
\hline 2 & Educational status & & \\
\hline $\mathrm{a}$ & No formal education & 24 & 16 \\
\hline $\mathrm{b}$ & Primary education(1-7) & 56 & 37.3 \\
\hline $\mathrm{C}$ & High school(8-10) & 45 & 30 \\
\hline $\mathrm{d}$ & PUC & 13 & 8.7 \\
\hline $\mathrm{e}$ & Graduate level and above & 12 & 8 \\
\hline 3 & Religion & & \\
\hline $\mathrm{a}$ & Hindu & 87 & 58 \\
\hline$b$ & Muslim & 46 & 30.7 \\
\hline c & Christian & 17 & 11.3 \\
\hline 4 & Average monthly income & & \\
\hline $\mathrm{a}$ & $<5000 /-$ & 74 & 49.3 \\
\hline $\mathrm{b}$ & $5000-10000 /-$ & 45 & 30 \\
\hline C & $>10000 /-$ & 31 & 27 \\
\hline 5 & Occupation & & \\
\hline$a$ & Working woman & 52 & 34.7 \\
\hline $\mathrm{b}$ & Non-working woman & 98 & 65.3 \\
\hline 6 & Type of the family & & \\
\hline A & Joint family & 77 & 51.4 \\
\hline B & Nuclear family & 73 & 48.6 \\
\hline 7 & Gravida & & \\
\hline A & Primigravida & 72 & 48 \\
\hline B & Multigravida & 70 & 46.7 \\
\hline C & Grandmultipara & 8 & 5.3 \\
\hline 8 & Mode of delivery & & \\
\hline A & Vaginal delivery & 52 & 71.24 \\
\hline$B$ & Caesarean delivery & 21 & 28.76 \\
\hline 9 & Gestational weeks & & \\
\hline A & $<12$ weeks & 19 & 12.6 \\
\hline$B$ & $13-28$ & 50 & 32.7 \\
\hline C & $29-40$ & 81 & 54.7 \\
\hline
\end{tabular}

Section 2: Occurrence of selected risk factors of pregnancy Frequency and percentage distribution of risk factors

\begin{tabular}{|c|c|c|c|}
\hline RISK STATUS & RISK FACTORS & FREQUENCY & PERCENTAGE \\
\hline \multicolumn{4}{|c|}{ BIOPHYSICAL RISK FACTORS } \\
\hline \multicolumn{4}{|c|}{ Age } \\
\hline No Risk & 1. $19-29 \mathrm{yrs}$ & 116 & 77.3 \\
\hline \multirow[t]{2}{*}{ At Risk } & $2.30-35$ yrs & 28 & 18.7 \\
\hline & 3. $<19$ or $>35$ yrs & 6 & 4 \\
\hline \multicolumn{4}{|c|}{ Height } \\
\hline No Risk & 1. $>150 \mathrm{~cm}$ & 126 & 84 \\
\hline \multirow[t]{2}{*}{ At Risk } & $2.145-150 \mathrm{~cm}$ & 20 & 13.3 \\
\hline & 3. $<145 \mathrm{~cm}$ & 4 & 2.7 \\
\hline
\end{tabular}




\begin{tabular}{|c|c|c|c|}
\hline RISK STATUS & RISK FACTORS & FREQUENCY & PERCENTAGE \\
\hline \multicolumn{4}{|c|}{ Present pregnancy gestation } \\
\hline No Risk & 1. Single & 146 & 96.7 \\
\hline \multirow[t]{2}{*}{ At Risk } & 2. Twins & 4 & 2.7 \\
\hline & 3. Triplets and above & 0 & 0 \\
\hline \multicolumn{4}{|c|}{ Pulmonary disease } \\
\hline No risk & 1. No history of pulmonary disease & 143 & 95.4 \\
\hline \multirow[t]{2}{*}{ At risk } & 2. Previous history of pulmonary disease but treated & 5 & 3.3 \\
\hline & 3. Present history of pulmonary disease and on treatment & 2 & 1.4 \\
\hline \multicolumn{4}{|c|}{ Cardiac disease } \\
\hline No risk & 1. No history of cardiac disease & 146 & 97.3 \\
\hline \multirow[t]{2}{*}{ At risk } & 2. Cardiac disease present and treated & 3 & 2 \\
\hline & 3. cardiac disease present and not treated & 1 & .7 \\
\hline \multicolumn{4}{|c|}{$\begin{array}{ll}\text { Blood pressure } \\
\end{array}$} \\
\hline No risk & 1. $120 / 80 \mathrm{~mm}$ of $\mathrm{Hg}$ & 131 & 87.3 \\
\hline \multirow[t]{2}{*}{ At risk } & 2. $>120 / 80$ but $<140 / 90 \mathrm{~mm}$ of Hg & 9 & 6 \\
\hline & 3. $>140 / 90 \mathrm{~mm}$ of $\mathrm{Hg}$ & 10 & 6.7 \\
\hline \multicolumn{4}{|c|}{ Family history of blood pressure } \\
\hline No risk & 1. No family history of hypertension or pregnancy induced hypertension & 110 & 73.3 \\
\hline \multirow[t]{2}{*}{ At risk } & 2. Family history of hypertension & 35 & 23.3 \\
\hline & 3. Family history of pregnancy induced hypertension & 5 & 3.4 \\
\hline \multicolumn{4}{|c|}{ Random blood sugar level } \\
\hline No risk & $1.80-119 \mathrm{mg} / \mathrm{dl}$ & 130 & 86.7 \\
\hline \multirow[t]{2}{*}{ At risk } & 2. $120-200 \mathrm{mg} / \mathrm{dl}$ & 17 & 11.3 \\
\hline & 3. $>200 \mathrm{mg} / \mathrm{dl}$ & 3 & 2 \\
\hline \multicolumn{4}{|c|}{ Diabetes } \\
\hline No risk & 1. No history of diabetes & 137 & 91.3 \\
\hline \multirow[t]{2}{*}{ At risk } & 2. Diabetes under control & 11 & 7.3 \\
\hline & 3. Diabetes not under control & 2 & 1.3 \\
\hline \multicolumn{4}{|c|}{ Family history of diabetes mellitus or previous overweight baby } \\
\hline No risk & 1. No family history of diabetes mellitus or previous overweight baby & 127 & 84.7 \\
\hline \multirow[t]{2}{*}{ At risk } & 2. Family history of diabetes mellitus & 20 & 13.3 \\
\hline & 3. Both family history of diabetes mellitus and overweight baby & 3 & 2 \\
\hline \multicolumn{4}{|c|}{ Renal disease } \\
\hline No risk & 1. No history of any renal disease & 145 & 96.7 \\
\hline \multirow[t]{2}{*}{ At risk } & 2. Previous history of renal disease but treated & 5 & 3.3 \\
\hline & 3. Present history of renal disease and on treatment & 0 & 0 \\
\hline \multicolumn{4}{|c|}{$\begin{array}{ll}\text { Fibroids } \\
\end{array}$} \\
\hline No risk & 1. No history of uterine fibroids & 144 & 96 \\
\hline \multirow[t]{2}{*}{ At risk } & 2. History of asymptomatic fibroids & 4 & 2.7 \\
\hline & 3. History of symptomatic fibroids & 2 & 1.3 \\
\hline \multicolumn{4}{|c|}{ Hemoglobin concentration } \\
\hline No risk & 1. $>10 \mathrm{gm} / \mathrm{dl}$ & 129 & 86 \\
\hline At risk & 2. 7-10gm/dl & 18 & 12 \\
\hline & 3. $<7 \mathrm{gm} / \mathrm{dl}$ & 3 & 2 \\
\hline & Abortions & & \\
\hline No risk & 1. No abortions & 120 & 80 \\
\hline At risk & 2. 1-2 abortions & 27 & 18 \\
\hline & 3. $>2$ abortions & 3 & 2 \\
\hline & Vaginal bleeding & & \\
\hline No risk & 1. No vaginal bleeding & 142 & 94.7 \\
\hline At risk & 2. Mild bleeding but controlled & 6 & 4 \\
\hline & 3. Severe bleeding treated with blood transfusion & 2 & 1.3 \\
\hline
\end{tabular}




\begin{tabular}{|c|c|c|c|}
\hline RISK STATUS & RISK FACTORS & FREQUENCY & PERCENTAGE \\
\hline \multicolumn{4}{|c|}{ Infectious disease } \\
\hline No risk & 1. No history of any infectious disease & 148 & 98.7 \\
\hline \multirow[t]{2}{*}{ At risk } & 2. Previous History of infectious disease and treated & 2 & 1.3 \\
\hline & 3. Present history of infectious disease on treatment & 0 & 0 \\
\hline \multicolumn{4}{|c|}{ Seizures } \\
\hline No risk & 1. No history of seizures & 146 & 97.3 \\
\hline \multirow[t]{2}{*}{ At risk } & 2. Seizures but controlled & 4 & 2.7 \\
\hline & 3. Seizures but not controlled & 0 & 0 \\
\hline \multicolumn{4}{|c|}{ Hypothyroidism or hyperthyroidism } \\
\hline Norisk & 1. No history of hypothyroidism or hyperthyroidism & 146 & 97.3 \\
\hline \multirow[t]{2}{*}{ At risk } & 2. hypothyroidism or hyperthyroidism but under control & 4 & 2.7 \\
\hline & 3. Not under control & 0 & 0 \\
\hline \multicolumn{4}{|c|}{ Pregnancy related complications or inherited disorders } \\
\hline No risk & 1. No family history of inherited disorders or pregnancy related complications & 143 & 95.3 \\
\hline \multirow[t]{2}{*}{ At risk } & 2. Family history of inherited disorders or pregnancy related complications & 6 & 4 \\
\hline & 3. Family history of both inherited disorders and pregnancy related complications & 1 & .7 \\
\hline \multicolumn{4}{|c|}{ Antenatal visit } \\
\hline No risk & 1. Regular antenatal visit & 143 & 95.3 \\
\hline \multirow[t]{2}{*}{ At risk } & 2. Minimum one antenatal visit per trimester & 7 & 4.7 \\
\hline & 3. No antenatal visit & 0 & $\mathrm{O}$ \\
\hline \multicolumn{4}{|c|}{ PSYCHOSOCIAL FACTORS } \\
\hline \multicolumn{4}{|c|}{$\begin{array}{l}\text { Psychiatric disorders } \\
\end{array}$} \\
\hline No risk & 1. No history of psychiatric disorders & 145 & 96.7 \\
\hline \multirow[t]{2}{*}{ At risk } & 2. History of psychiatric disorders but treated & 4 & 2.7 \\
\hline & 3. History of psychiatric disorders but not treated & 1 & .7 \\
\hline \multicolumn{4}{|c|}{ Smoking or alcohol } \\
\hline No risk & 1. No smoking or alcohol & 0 & 0 \\
\hline \multirow[t]{2}{*}{ At risk } & 2. Smoking or alcohol & & \\
\hline & 3. Smoking and alcohol & 0 & 0 \\
\hline \multicolumn{4}{|c|}{ Access to health care facilities } \\
\hline No risk & 1. Adequate access to health care facilities(within $1 \mathrm{~km}$ ) & 140 & 96.3 \\
\hline \multirow[t]{2}{*}{ At risk } & 2. Limited access to health care facilities (less than or equal to $5 \mathrm{~km}$ ) & 7 & 4.7 \\
\hline & 3. Very limited access to health care facilities(more than $5 \mathrm{~km}$ ) & 3 & 2 \\
\hline \multicolumn{4}{|c|}{$\begin{array}{ll}\text { Family support } \\
\end{array}$} \\
\hline No risk & 1. Good family support & 145 & 96.7 \\
\hline \multirow[t]{2}{*}{ At risk } & 2. Support from spouse or family only & 5 & 3.3 \\
\hline & 3. No support from family and spouse & 0 & 0 \\
\hline \multicolumn{4}{|c|}{ ENVIRONMENTAL FACTORS } \\
\hline \multicolumn{4}{|c|}{$\begin{array}{l}\text { Radiation or pesticides } \\
\end{array}$} \\
\hline No risk & 1. No exposure to radiation or pesticides & 146 & 97.3 \\
\hline \multirow[t]{2}{*}{ At risk } & 2. Exposure to radiation or pesticides & 4 & 2.7 \\
\hline & 3. Exposure to radiation and pesticides & 0 & 0 \\
\hline \multicolumn{4}{|c|}{ Industrial pollutants } \\
\hline No risk & 1. No exposure to any industrial pollutants & 147 & 98 \\
\hline \multirow[t]{2}{*}{ At risk } & 2. Limited Exposure to industrial pollutants & 3 & 2 \\
\hline & 3. Exposure to industrial pollutants & 0 & 0 \\
\hline
\end{tabular}

\section{Discussion :}

The study findings revealed that increased maternal age, short stature, increased blood pressure, abortion, decreased hemoglobin and GDM were the most prevalent risk factors in the study sample. Considering increased 
complications during pregnancy; the most frequent complications were gestational diabetes (12.7\%) and preeclampsia $(10.1 \%)^{2}$. The finding of short stature as a risk is congruent with a population-based study performed in Israel during 2000-2004 .The study revealed that of 159,210 deliveries occurred, 5822 (3.65\%) were of patients with short stature. Patients with short stature had statistically significant higher rates of CS compared with patient's $\geqslant 155 \mathrm{~cm}$ ( $21.3 \%$ versus $11.9 \%)$. Higher rates of intrauterine growth restriction (3.2\% versus $1.9 \%)$, premature rupture of membranes (7.1\% versus $5.6 \%)$, labor dystocia (6.1\% versus 3.5\%), mal-presentations (7.6\%

\section{References:}

1. Neely Melanie Curtis. Breastfeeding experiences of mothers using tele health at one and four weeks postpartum. Journal of BSN Honors Research. 2010; 1(1)

2. Stamp G E.Casanova H T.A breast feeding study in a rural population in South Australia. Rural and Remote Health. 2006; 6:495

3. Margaret. Schmied Virginia. Sheehan Athena. An exploration of the relationship between postnatal distress and maternal role attainment, breast feeding problems and breast feeding cessation in Australia. Midwifery.2007; 23(1):66-76

4. E R.Giugliani. Common problems during lactation and their management. J Pediat 2004; 80(5)147-54. versus 6.1\%), and cephalopelvic disproportion $(0.9 \%$ versus $0.3 \%)^{13}$ was also found ${ }^{3}$.

\section{Conclusion :}

The goal of maternity care is healthy pregnancy with a physically safe and emotionally satisfying outcome for mother, infant and family. The study findings revealed that women are at risk for the pregnancy. Although health information alone is insufficient to change behaviors, it may contribute to more informed decisions which necessitates the health professionals to be responsible to educate the women regarding the risk factors of pregnancy thus to attain the goal of maternity care 